\title{
A Nano Capacitor with Graphene Electrodes and Methane - (h-BN) Insulator
}

\author{
FARROKH ROYA NIKMARAM \\ Department of Chemistry, Faculty of Science, \\ Yadegar-e-Imam Khomeini (RAH) Shahre Rey Branch, Islamic Azad University, Tehran, Iran \\ ${ }^{*}$ Corresponding author E-mail: nikmaram87@yahoo.com \\ http://dx.doi.org/10.13005/ojc/320120
}

(Received: January 12, 2016; Accepted: February 13, 2016)

\begin{abstract}
Methan has a large potential to adsorb and diffuse among h-BN and graphene surfaces as the suitable dielectric. With this background the nanoscale dielectric capacitors have been widely studied due to their ability to store a high amount of energy. In this research, I have modeled one which is composed of two graphene layers including insulating medium of a h-BN layers which are filed out (Methane)n, $m\{n=m=7)$. It has been indicated that the Methane molecule is the suitable gas for hetero-structures of the $\mathrm{G} /(\mathrm{h}-\mathrm{BN})$-(Methane)7,7/G capacitor compared to those nonpolar gases of 3 atoms. The quantum and coulomb blocked effects of h-BN/graphene including different numbers of Methane for multi dielectric properties of diûerent thicknesses have been studied. We have shown that Quantum effect has appeared in a small thickness of capacitor due to number of layers and Methane atoms.
\end{abstract}

Key words: Capacitor, Graphene, Boron nitride sheet, Coulomb blockade, Methane properties.

\section{INTRODUCTION}

The $s p^{2}$-hybridized B-N and C-C bonding has very similar characteristics in mechanics, while great difference in optics and electronics. Essentially, the similar points result from the close positions of $B$, $\mathrm{C}$ and $\mathrm{N}$ in the Mendeleev Table, while the different ones result from the heterogeneous atoms. The bonding orbit $\mathrm{p}$ of $\mathrm{B}-\mathrm{N}$ is mainly dominated by $2 p$ orbit of $\mathrm{N}$, while $2 p$ orbit of $\mathrm{B}$ contributes mostly to the antibonding orbit $p^{*}$.
The discovery of graphene in $2004^{1}$, an atomically thin two-dimensional (2D) graphitic crystal, offers new opportunities to nano electronics and nano materials and has stimulated worldwide enthusiasm for the research of $2 \mathrm{D}$ nano materials ${ }^{2}$.

Graphene has a relatively flexible molecular structure due to its electronic properties. It can be chemically fitted on top of a deposit metal atom ${ }^{1-4}$, a deposit molecule can be fitted on its top ${ }^{2}$, nitrogen and boron can be incorporated in its structure ${ }^{3}$ and 
can have doped atom inside its sheet. This structure can be used in a wide area of researches such as electronics, capacitor, superconductors, batteries and diodes. The considerable characteristic of graphene is that it is a Dirac solid, with the electron energy being linearly dependent on the wave vector near the vertices of the hexagonal Brillouin zone ${ }^{4}$. A complete history for the rising of graphene has been given by Geim and Novoselov 5 .

Graphene has studied in two-dimensional nanostructures, including carbon and hydrogen atoms with $\mathrm{sp}^{2}$-hybrid lattice structures. Graphene has a structure analogous to benzene and polycyclic aromatic hydrocarbons and $\mathrm{sp}^{2}$ hybrid, the chemistries of the compounds can be thought to be similar, graphene has many novel properties, such as high surface area, excellent electrical conductivity and electron mobility at room temperature, and has unique thermal and mechanical properties recent investigations have demonstrated that graphene may find tremendous applications in many areas ${ }^{3,4}$.

The nanoscale capacitors have recently developed and achieved properties which are premier to other systems of energy storage. The nanoscale dielectric capacitors (NDC) consist of two metallic graphene layers separated by insulating $\mathrm{h}-\mathrm{BN}$ thin layers which have been successfully used to simulate structures of graphene/h-BN/graphene ${ }^{1-4}$.

Both theoretical and experimental studies on metallic graphene were focused on understanding the dielectric properties of these structures and forming thin layers that can bestead as charge holding plates ${ }^{2,4}$. Furthermore it was shown that graphene can preserve current densities six order of magnitude larger than copper ${ }^{5}$. It has been theoretically ${ }^{6}$ and experimentally ${ }^{7}$ shown that $\mathrm{h}-\mathrm{BN}$ layers of any thickness can be grown on graphene layers and it is also possible to flourish perpendicular carbon on top of the sheets ${ }^{8}$ and is important material for adsorption ${ }^{9-14}$.

In this study, we consider a NDC model of G/ (h-BN)m-(Methane)7,7/G capacitor composed with (h- BN) sheets Methane gas as an insulator using ab initio calculations within the density functional theory (DFT) and extended-huckel. Our nanoscale capacitor model is composed of a few hexagonal h-BN layers, which are stacked between two metallic graphene sheets as can be seen in scheme1.

The h-BN belongs to the same family of stacked hexagonal materials as graphene, which consists of atomically thin sheets held together by weak van der Waals forces. Its intra-layer network consists of strong covalent bonds, whose partial ionic character turn the system into an insulator.

Multilayers of $\mathrm{h}-\mathrm{BN}$ are wide band gap insulators which can vail as a dielectric material between metal-doped graphene layers. In addition, the sheets that are lattice matched to graphene allow one to attain sturdy and high accuracy nanoscale spacing between two parallel metallic graphene plates, which can be set to favorable values.

Monajjemi and coworkers have studied of nanotube carbon and graphene as electronic devices such as LiBTs, capacitors, diode, various transistor and biological sensors both in solvent and vacuum media, widely ${ }^{15-131}$

Since the thickness of separation of the capacitors can be less than 15 angstrom, the stored energy has to be calculated from the ûrst principles. However, the available ûrst-principle methods allow us to treat the distribution of only one kind of excess positive or negative charge at a time in the same situation. Therefore, the main problem in this kind of calculations is related to separating positive and negative charges on the plates of electrodes in a capacitor. In our model, the charge carriers are electrons themselves; while they exist in excess in one plate, they are unloaded from the other one.

\section{Theoretical model}

In this model, a small capacitor is made by creating an insulating layer of (h-BN) between two metal-doped graphene surfaces. We assume that the capacitor electrodes carry $\pm Q$ charges from one electrode towards the opposite side. So the initial energy stored in the electr ${ }_{E_{i}}=\frac{Q^{2}}{2 C}$
field between the capacitor plates is given by

One of the most fundamental effects in nano electronics is related to the significant change 
in the energy when the electrons are transferred into a nanoscopic material region such as quantum dot, which results in what is known as "Coulomb Blockade".

By letting the electrons tunnel through the insulating layer from the negative terminal to the positive terminal, such that the charge $\left(Q+{ }^{\prime} q_{e}\right)$ resides on the top plate and (" $\mathrm{Q}-\mathrm{Hq}_{\mathrm{e}}$ ) resides on the bottom plate, the stored energy in this situation is $\operatorname{now}^{E_{f}}=\frac{(Q+\Delta q)^{2}}{2 c}$.

Although the charge itself is quantized, the charge on the capacitor plates is polarized and not quantized. The energy cannot be stored in the capacitor plates until a single electron tunnels through the insulator h-BN layers from the negative terminal to the positive terminal where $\ddot{\Delta} E_{s}=E_{f}-E_{i}=\frac{\Delta q\left(Q^{\prime}+\frac{\Delta q}{2}\right)}{c}$ ergy can be indicated as: large voltage is in the range of $-\frac{\Delta \bar{q}}{2 C}<\Delta V<+\frac{\Delta \bar{q}}{2 C}$

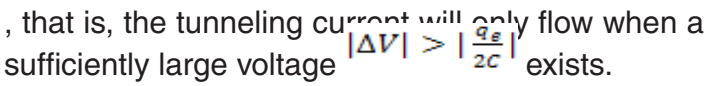

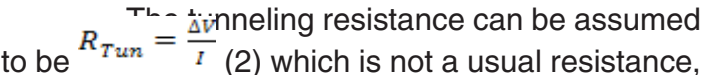
however, theoretically allowing electrons to cross the insulating junction as discrete occurrences where "l" is the resulting current due to the tunneling effect. Tunneling resistance is not an usual resistance, but an imaginary one, allowing the electrons to cross the

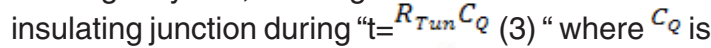
the quantum capacitance and ${ }^{t}$ is a characteristic time associated with tunneling events which is considered to be the approximate lifetime of the energy state of the electrons (on one side of the barrier).

The $R_{\text {Tun }}$, should be finite and not too big, so that tunneling can practically take place. In this case, the charge is said to be well quantized and the capacitor is considered to be a tunnel junction.

In this work we have calculated the $\mathrm{R}_{\text {Tun }}$ for various capacitors (including graphene electrodes composed with ( $\mathrm{h}-\mathrm{BN}$ ) sheets and Methane gas as the insulators $\}$ as a function of the thickness of $(\mathrm{h}-\mathrm{BN})_{\mathrm{n}}$ and difference in potential energy barrier between the capacitor electrodes (Via the uncertainty relationship between time and energy, $\Delta E \Delta t \geq \frac{\hbar}{2} \rightarrow R_{\text {Tun }} \geq \frac{h}{2 \pi q_{\varepsilon}^{2}}(4)$

When the quantum well descends below the Fermi level, the electrons start to be accommodated in this quantum well and the excess electrons in the graphene layers become sensitive to charge spilling into the vacuum space of capacitor.

Table 1: Charges, HOMO, LUMO and Gap energy of the capacitor including $7+7=14$ molecules of Methane

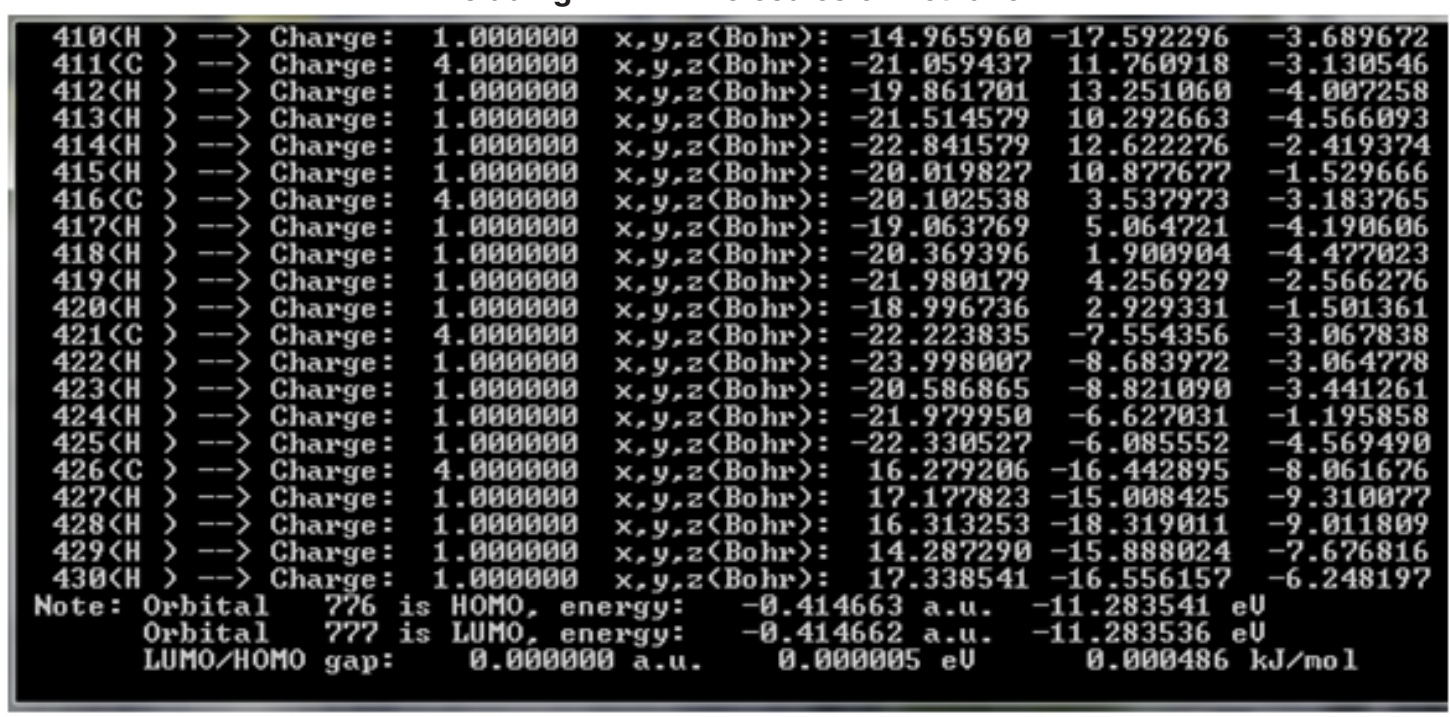


Ajayan and his coworkers have suggested ${ }^{7}$ that the observed abnormal increase in capacitance with decreasing size is due to the negative quantum capacitance for nano-systems. This eûect arises from many-body interactions since the chemical potential of the electrons decreases with the increase of electron density. A few studies have reported different aspects of quantum capacitance; of particular importance are the study of Maccuci whom has reported a capacitance of radial quantum dots, Wang et al., on capacitance of atomic junctions ${ }^{132}$ and another study on non-linear quantum capacitance ${ }^{133}$.

The QM component is a development of the density of states of the metal electrodes, and their Thomas-Fermi screening lengths. Hence the hybrid capacitanc ${ }^{-1}=1_{1} \cdots c_{1}$ itor architecture is given as: $\overline{c_{\text {net }}}=\frac{1}{c_{Q_{1}}}+\frac{1}{c_{Q_{2}}}+\frac{1}{c_{g}}$ (5) Where $C_{\text {net }}$ is the net capacitance of the nano-capacitor, ${ }^{C_{g}}$ is the classical (geometric) capacitance, $C_{Q_{1}}$ and $C_{Q_{2}}$ are the quantum capacitances, due to the density of states of the $(\mathrm{Li}, \mathrm{Be}, \mathrm{B})$, doped graphene electrodes $M_{1} G$, and $M_{2} G$, respectively.

\section{Computational details}

In this study, we have mainly focused on getting the results from DFT methods such as m06,
m06-L, and extended-Huckel for the non-bonded interaction of $\mathrm{G} /(\mathrm{h}-\mathrm{BN})$-(Methane)n/G which are monotonous through the comparison between different situations. The m062x, m06-L, and m06-HF are new methane hybrid DFT functional with a good correspondence in non-bonded calculations and are useful for calculating the energies of the distance between two fragments in a capacitor. The double $æ$-basis set with polarization orbitals were used for doped graphene atoms while single $æ$-basis sets with polarization orbitals were employed for the h-BN layers. Calculations were performed using Gaussian 09 and GAMESS-US packages.

For a non-covalent interaction, B3LYP is unable to describe van der Waals interactions ${ }^{134,135}$ in capacitor systems by medium-range interactions, such as the interactions of two electrodes and dielectric sheets. The lack of ability of B3LYP and most other popular functional to correctly describe medium-range of exchange and correlation energy limits their applicability for distant non-bonded systems of two electrodes and dielectric thickness in a capacitor. Moreover, some recent studies have shown that inaccuracy for the medium-range exchange energies lead to large systematic errors in the prediction of molecular properties ${ }^{136}$.

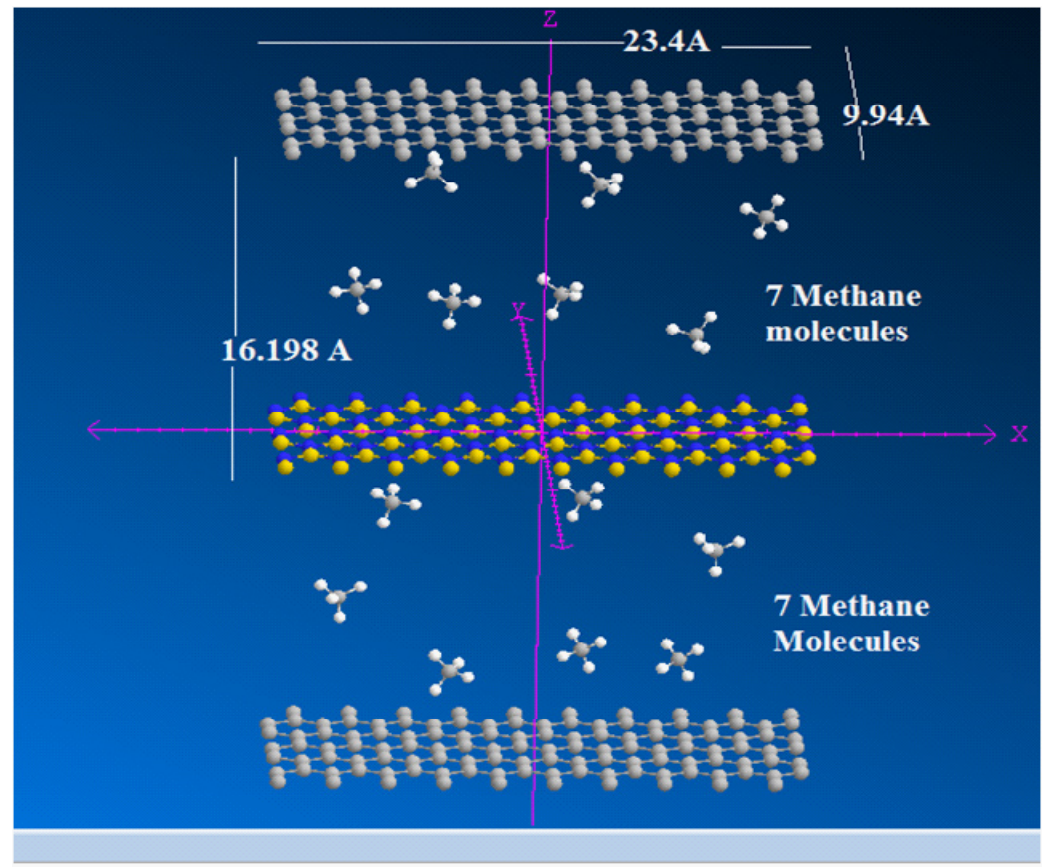

Fig. 1: The dimension of $\mathrm{G} /(\mathrm{h}-\mathrm{BN})$ 
A monolayer of graphene was optimized and allowed to relax to its minimum energy structure. It contains 45 atoms. After relaxation, all C-C-C angles and $\mathrm{C}-\mathrm{C}$ bond were calculated to be around $120^{\circ}$ and $1.421 \AA$, respectively, which are in good agreement with reported values ${ }^{137}$.

Geometry optimizations and electronic structure calculations have been carried out using the DFT approach which is based on an iterative solution of the Kohn-Sham equation ${ }^{138}$ of the density functional .
The Perdew-Burke-Ernzerhof ${ }^{139}$ exchangecorrelation (XC) functional of the generalized gradient approximation (GGA) is adopted. The dimension of capacitor has been set via three dimensions as $5.655 \times 12.306 \times 15.037 \AA$ and the sheets are separated by various distances along the perpendicular direction to avoid interlayer interactions (scheme1). During all of the calculation processes, the partial occupancies were considered by using the Bloch corrections ${ }^{140}$.
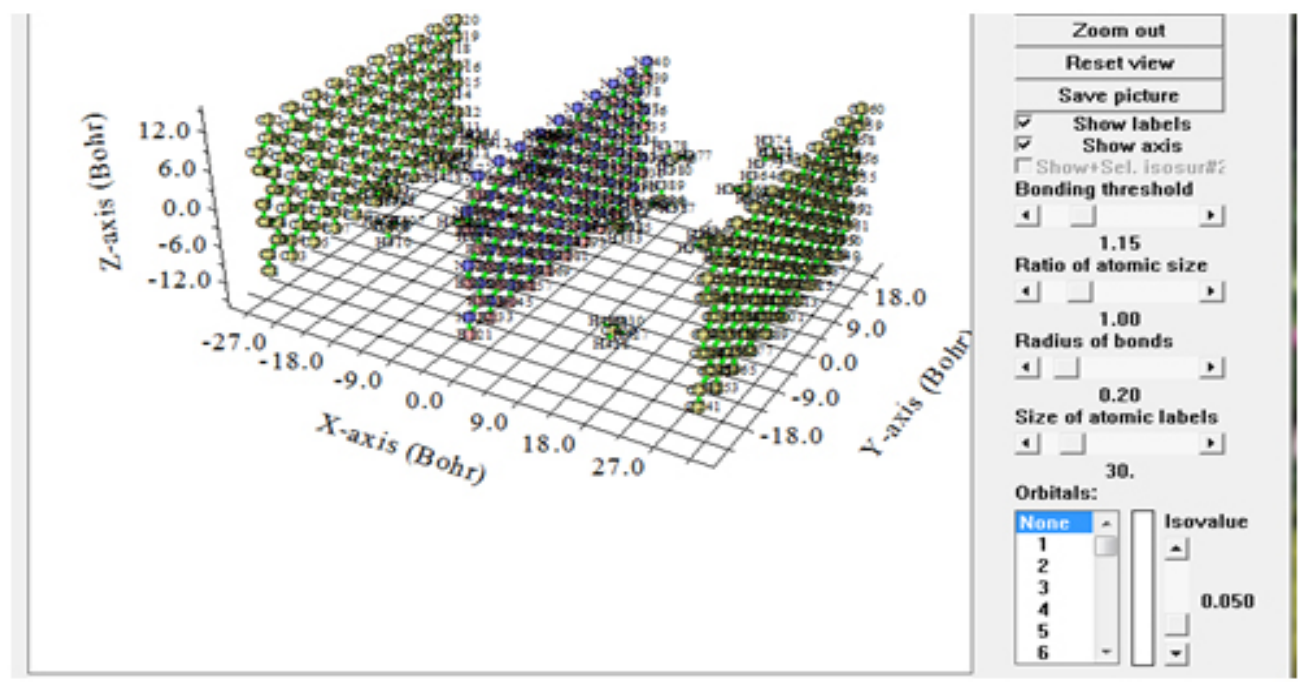

Fig. 2: The Capacitour versues 3 axis (Bohr)

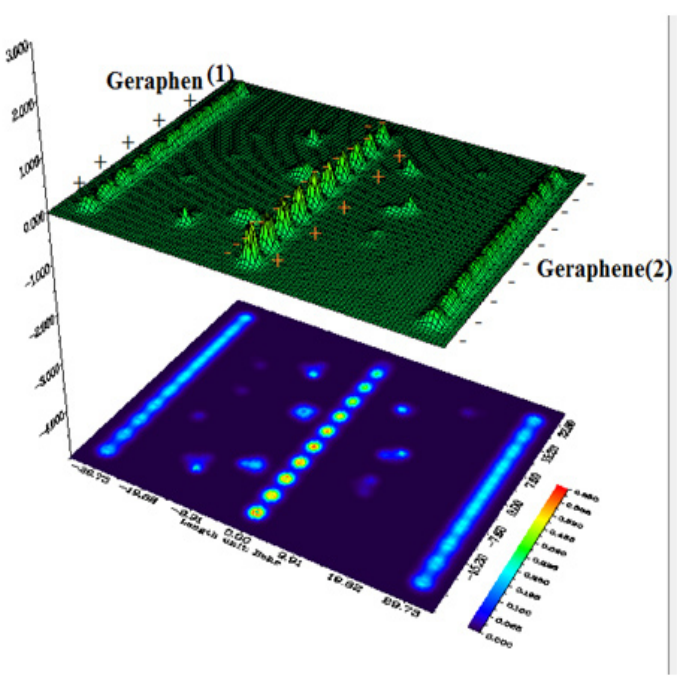

Fig. 3: The electron density and Charges localization of Capacitor with projection
The charge calculation methods based on molecular electrostatic potential (MESP) fitting are not well-suited for treating larger systems where some of the innermost atoms are located far away from the points at which the MESP is computed. In such a condition, variations of the innermost atomic charges will not lead to significant changes of the MESP outside of the molecule, meaning that the accurate values for the innermost atomic charges are not well-determined by MESP outside of the molecule. The representative atomic charges for molecules should be computed as average values over several molecular conformations. Although infinite graphene sheets are intrinsically metallic, our BG system exhibits an increase in the metallic properties. The interaction energy for capacitor was calculated in all items as indicated in the equation 9: 
$\Delta E_{S}(\mathrm{eV})=\left\{E_{\mathrm{G} /(\mathrm{h}-\mathrm{BN}) \mathrm{m}-(\mathrm{He}) \mathrm{n} / \mathrm{G}}-\left(2 E_{G}+E_{(h-B N) m}+E_{(\mathrm{He}) n}\right)\right\}$

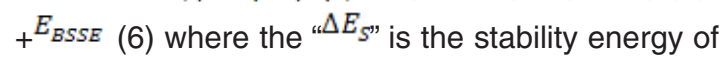
capacitor.
The electron density (Both of Gradient norm \& Laplacian), value of orbital wave-function, electron spin density, electrostatic potential from nuclear atomic charges, electron localization function (ELF), localized orbital locator (LOL defined by Becke \&

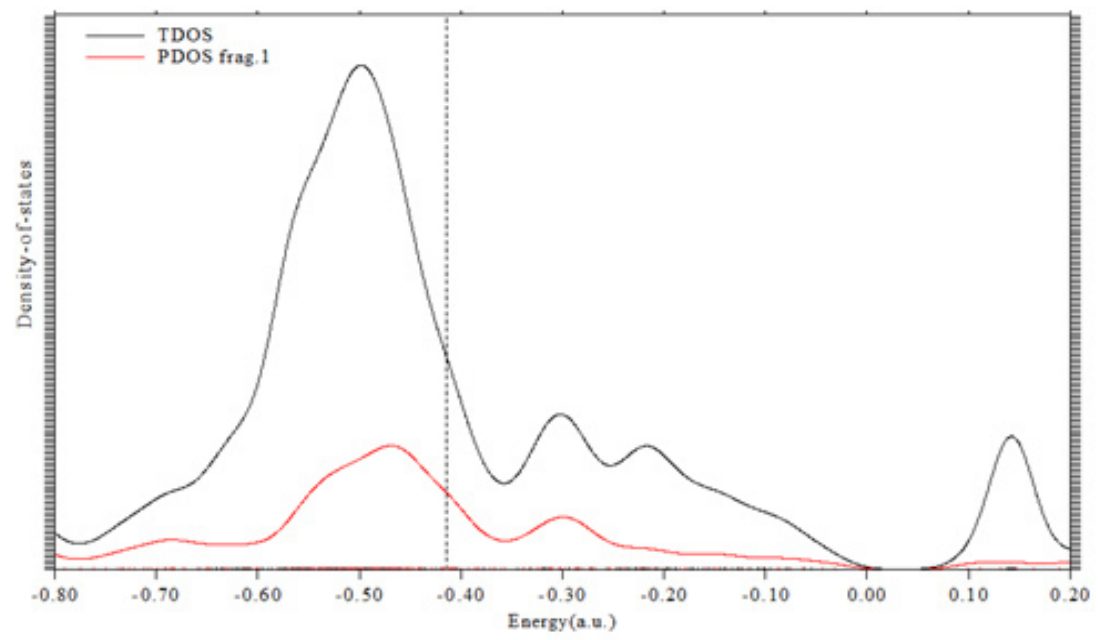

Fig. 4: The electron density and TDOS, PDOS, for three fragment for the G/(h-BN)( Methane) /G capacitor

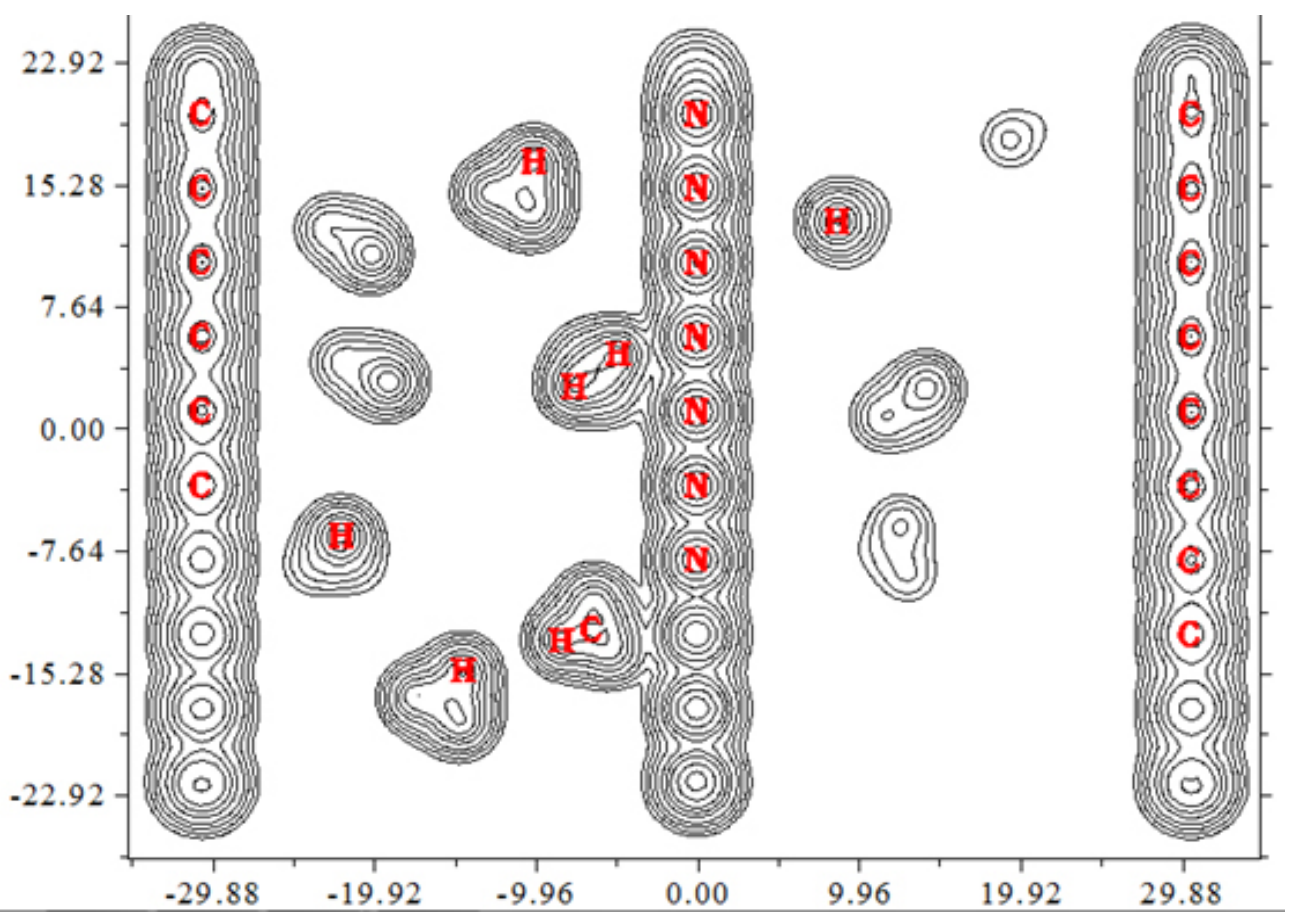

Fig. 5: Electron density of system with projection and counter map diagram 
Tsirelson), total electrostatic potential (ESP), as well as the exchange-correlation density, correlation hole and correlation factor, and the average local ionization energy.

\section{RESULTS AND DISCUSSION}

Horizontal boron nitride ( $\mathrm{h}-\mathrm{BN}$ ) in nature is an ideal electrical insulator that can be polarized by applying an external electric ûeld; the number of $\mathrm{h}-\mathrm{BN}$ layers between the graphene plates has been calculated and optimized as a suitable simulation of dielectrics. Furthermore, graphene and h- BN are two well-known single layer honeycomb structures, so the proposed model can be easily fabricated.

In this study, BN was chosen as a dielectric since it is an excellent spacer with a lattice constant close to that of graphene. We speciûcally studied the dielectric properties of $\mathrm{G} /(\mathrm{h}-\mathrm{BN})$ (Methane) /G, including the number of $\mathrm{h}-\mathrm{BN}$ respectively.

Similar to graphene, the anisotropic binding of $\mathrm{h}$-BN allows for the formation of various layered structures. Long-range interlayer interactions play a dominant role in characterizing the structural and mechanical properties of these systems and hence their performance in the simulation of our capacitor models.

The $s p^{2}$-hybridized B-N and $\mathrm{C}-\mathrm{C}$ bonding has very similar characteristics in mechanics, while great difference in optics and electronics. Essentially, the similar points result from the close positions of $\mathrm{B}$, $\mathrm{C}$ and $\mathrm{N}$ in the Mendeleev Table, while the different ones result from the heterogeneous atoms. The bonding orbit $\mathrm{p}$ of $\mathrm{B}-\mathrm{N}$ is mainly dominated by $2 p$ orbit of $\mathrm{N}$, while $2 p$ orbit of $\mathrm{B}$ contributes mostly to the antibonding orbit $\mathrm{p}^{*}$. This implies a significant charge transfer from $\mathrm{B}$ to $\mathrm{N}$, around 0.4 electrons. In contrary, there is no significant electron transfer in $\mathrm{C}-\mathrm{C}$ bonding so the $\mathrm{B}-\mathrm{N}$ bonding is partially ionic; while $\mathrm{C}-\mathrm{C}$ bonding is covalent (This also leads to the different electron band structure).

The values of the distances between graphene layers capping h-BN layers, dielectric constants of the layered $h-B N$ sheets $(k)$, magnitude of the charges on the graphene plates, electrostatic properties using the SCF density, fitting point charges to electrostatic potential charges from ESP fit, the stability energy of capacitor $(\mathrm{eV})$, various capacitances including the net capacitance and the potential difference between two electrodes of graphene plates are listed in tables $\{1-2\}$.

Gap energy and kinetic energy, potential energy, Laplacian of electron density and density of all electrons are depended to various number of $\mathrm{He}$ in the table1 which it can be exhibited the proper situations for $\mathrm{He}$ and number of $\mathrm{h}$-BN plates in figs 1-5.

In addition, according to the electronic structures, we have considered two isolated graphene layers which are doped by these atoms in various distances. The atomic structures, interlayer spacing, relative positions of the layers and the cell parameters have been optimized.

Here we have considered the interlayer attraction using extended-Huckel force field for $\mathrm{h}-\mathrm{BN}$ to describe its interlayer interactions including $h-B N$ inter-layer potential, attractive components and the classical mono-polar electrostatic term that takes into account the partially ionic character of h-BN.

Table 2: The Charges of two graphene electrodes and the stability energy, difference potential, dielectric

\begin{tabular}{lcccc}
\hline $\begin{array}{l}\text { Methane } \\
\text { number }\end{array}$ & $\Delta E_{5}(e V)$ & $|\Delta q|=\sum_{i=1}^{N}\left(q_{G_{1}}-q_{G_{2}}\right)$ & $\Delta V=\sum_{i=1}^{45}\left(E P_{G_{1}}-E P_{G_{2}}\right)$ & $\begin{array}{c}\text { Dielectric } \\
\text { constant(K) }\end{array}$ \\
\hline 1,1 & 0.23 & 0.12 & 1.44 & 1.1 \\
2,2 & 0.32 & 0.15 & 2.34 & 1.5 \\
5,5 & 0.45 & 0.18 & 1.56 & 1.8 \\
7,7 & 0.57 & 0.23 & 1.99 & 1.9 \\
\hline
\end{tabular}


For long distances of dielectric thickness, the classical capacitance rule of the " $C_{g} \propto \frac{1}{d}$ " is adaptable. This adaptability does not go for short distances, which is attributed to the quantum size effect. We identified the dielectric permittivity as a function of dielectric size through abinito calculations,

$C_{Q}(F) \times 10^{20}=\frac{\Delta q\left(Q+\frac{\Delta a}{2}\right)}{\Delta E_{S}} \quad C_{\text {net }}(F) \times 10^{20}=\frac{C_{g} C_{Q}}{C_{Q}+2 C_{g}} \quad$ and Quantum effect by $\dot{R_{T u n}(K \Omega) \gg \frac{\hbar}{\Delta_{E S F}^{2}}}$, using the molecular dynamic simulation.

\section{REFERENCES}

1. Novoselov, KS.; Geim, AK.; Morozov, SV.; Jiang, D.; Zhang, Y.; Dubonos. SV.; Grigorieva, IV.; Firsob, AA, Science, 2004, 306, 666-669

2. Zhengzong, S.; Dustin, K J.; Tour, JM, J Phys Chem Lett, 2011, 2:2425-2432

3. Nomura,K.; Ohta,H.; Ueda,K.; Kamiya,T.; Hirano,M.; Hosono,H, Science,2003 300 1269.

4. Huangz, M. H.; Mao, S.; Feick, H.; Yan, H.;Wu, Y.; Kind, H.; Webber, E.; Russo, R.; Yang, P, Science, 2001, (292):1897-1899

5. Geim, A.K. Science, 2009, $324,1530-$ 1534.

6. Özçelik, V.O.; Cahangirov, S.; Ciraci, S, Phys. Rev. $B$,2012, $85,235456$.

7. Liu, Z.; Song, L.; Zhao, S.; Huang, J.; Ma, L.; Zhang, J.; Lou, J.; Ajayan, P.M., Nano Lett. 11 2011, 2032-2037.

8. Ataca, C.; Ciraci, S, Phys. Rev. B , 2011, 83, 235417.

9. Sadegh,H.; Shahryari-ghoshekandi,R.; Agrawal, S.; Tyagi,I.; Asif,M, Gupta,V.K, Journal of Molecular Liquids, 2015, 206, 151158.

10. Gupta,V.K.; Tyagi,I.; Agrawal,S.; Sadegh,H.; Shahryari-ghoshekandi, R.; Yari,M.; Yousefinejat,O.; Journal of Molecular Liquids, 2015, 206, 126-136

11. Sadegh,H.; Shahryari-ghoshekandi,R.; Tyagi,I.; Agrawal,S.; Gupta,V.K, Journal of Molecular Liquids,2015, 207, 21-27.

12. Sadegh,H.: Zare,K.; Maazinejad,B.; Shahryarighoshekandi,R.; Tyagi,I.; Agrawal,S.; Gupta,V.K, Journal of Molecular Liquids, 2016,215, 221-228.

13. Zare,K.; Sadegh,H.; Shahryarighoshekandi,R.; Asif,M.; Tyagi,I.; Shilpi Agrawal, Gupta,V.K Journal of Molecular Liquids, 2016, 213, 345-350 (2016)

14. Omid Moradi, O.; Gupta,V.K.; Agrawal, S.;
Tyagi,I.; Asif,M.; Hamdy Makhlouf, A.S.; Sadegh,H.; Shahryari-ghoshekandi,R.; Journal of Industrial and Engineering Chemistry, 2015, 28, 294-301

15. Monajjemi, M.; Lee, V.S.; Khaleghian, M.; B. Honarparvar, B.; F. Mollaamin, F. J. Phys. Chem C. 2010, 114, 15315

16. Monajjemi, M. Struct Chem. 2012, 23,551580

17. Monajjemi, M.; Chegini, H.; Mollaamin, F.; Farahani, P. Fullerenes, Nanotubes, and Carbon Nanostructures. 2011, 19, 469-482

18. Monajjemi, M .; Afsharnezhad ,S.; Jaafari , M.R.; Abdolahi ,T.; Nikosade ,A.; Monajemi ,H.; Russian Journal of physical chemistry A, 2007, 2,1956-1963

19. Monajjemi, M.; Baei, M.T.; Mollaamin, F. Russian Journal of Inorganic Chemistry. 2008, 53 (9), 1430-1437

20. Monajjemi, M.; Rajaeian, E.; Mollaamin, F.; Naderi, F.; Saki, S. Physics and Chemistry of Liquids. 2008, 46 (3), 299-306

21. Monajjemi, M.; Boggs, J.E. J. Phys. Chem. A, 2013, 117, 1670 "1684

22. Mollaamin, F.; Monajjemi, M, Journal of Computational and Theoretical Nanoscience. 2012, 9 (4) 597-601

23. Monajjemi, M.; Khaleghian, M, Journal of Cluster Science. 2011, 22 (4), 673-692

24. Mollaamin, F.; Varmaghani, Z.; Monajjemi, M, Physics and Chemistry of Liquids. 2011, 49 318

25. Nafisi, S.; Monajemi, M.; Ebrahimi, S. Journal of Molecular Structure. 2004,705 (3) 35-39

26. Fazaeli, R.; Monajjemi, M.; Ataherian, F.; Zare, K. Journal of Molecular Structure: THEOCHEM.2002, 581 (1), 51-58

27. Monajjemi, M.; Razavian, M.H.; Mollaamin,F.; Naderi,F.; Honarparvar,B.; Russian Journal of Physical Chemistry A , 2008 , 82 (13), 2277 2285 
28. Monajjemi, M.; Seyed Hosseini, M.; Mollaamin, F. Fullerenes, Nanotubes, and Carbon Nanostructures. 2013, 21, 381-393

29. Monajjemi, M.; Faham, R.; Mollaamin, F. Fullerenes, Nanotubes, and Carbon Nanostructures, 2012 20, 163-169

30. Mollaamin, F.; Najafi, F.; Khaleghian, M.; Khalili Hadad, B.; Monajjemi, M. Fullerenes, Nanotubes, and Carbon Nanostructures, 2011 19, 653-667

31. Mollaamin, F.; Baei, MT.; Monajjemi, M.; Zhiani, R.; Honarparvar, B.; Russian Journal of Physical Chemistry A, Focus on Chemistry, 2008, 82 (13), 2354-2361

32. Monajjemi, M. Chemical Physics. 2013, 425, 29-45

33. Monajjemi, M.; Heshmat, M.; Aghaei, H.; Ahmadi, R.; Zare, K. Bulletin of the Chemical Society of Ethiopia, 2007, 21 (1)

34. Monajjemi, M.; Honarparvar, B. H. ; Haeri, H. ; Heshmat ,M.; Russian Journal of Physical Chemistry C. 2006, 80(1):S40-S44

35. Monajjemi, M.; Ketabi, S.; Amiri, A. Russian Journal of Physical Chemistry, 2006, 80 (1), S55-S62

36. Yahyaei, H.; Monajjemi, M.; Aghaie, H.; K. Zare, K. Journal of Computational and Theoretical Nanoscience. 2013, 10, 10, 2332-2341

37. Mollaamin, F.; Gharibe, S.; Monajjemi, M. Int. J. Phy. Sci, 2011, 6, 1496-1500

38. Monajjemi, M.; Ghiasi, R.; Seyed Sadjadi, M.A. Applied Organometallic Chemistry,2003, 17, 8, 635-640

39. Monajjemi, M.; Wayne Jr, Robert. Boggs, J.E. Chemical Physics. 2014, 433, 1-11

40. Monajjemi, M.; Sobhanmanesh, A.; Mollaamin, F. Fullerenes, Nanotubes, and Carbon Nanostructures, 2013, 21 47-63

41. Monajjemi, M.; Mollaamin, F. Journal of Computational and Theoretical Nanoscience, 2012, 9 (12) 2208-2214

42. Monajjemi, M.; Honarparvar, B.; Nasseri, S. M. .; Khaleghian M. Journal of Structural Chemistry. 2009, 50, 1, 67-77

43. Monajjemi, M.; Aghaie, H.; Naderi, F. Biochemistry (Moscow).2007, 72 (6), 652657

44. Ardalan, T.; Ardalan, P.; Monajjemi, M. Fullerenes, Nanotubes, and Carbon
Nanostructures, 2014, 22: 687-708

45. Mollaamin, F.; Monajjemi, M.; Mehrzad, J. Fullerenes, Nanotubes, and Carbon Nanostructures. 2014, 22: 738-751

46. Monajjemi, M.; Najafpour, J.; Mollaamin, F. Fullerenes, Nanotubes, and Carbon Nanostructures. 2013, 21(3), 213-232

47. Monajjemi, M.; Karachi, N.; Mollaamin, F. Fullerenes, Nanotubes, and Carbon Nanostructures, 2014, 22: 643-662

48. Yahyaei, H.; Monajjemi, M. Fullerenes, Nanotubes, and Carbon Nanostructures.2014, 22(4), 346-361

49. Monajjemi, M. Falahati, M.; Mollaamin, F.; Ionics, 2013, 19, 155-164

50. Monajjemi, M.; Mollaamin, F. Journal of Cluster Science, 2012, 23(2), 259-272

51. Tahan, A.; Monajjemi, M. Acta Biotheor, 2011, 59, 291-312

52. Lee, V.S.; Nimmanpipug, P.; Mollaamin, F.; Kungwan, N.; Thanasanvorakun, S..; Monajjemi, M. Russian Journal of Physical Chemistry A, 2009, 83, 13, 2288-2296

53. Monajjemi, M.; Heshmat, M.; Haeri, HH, Biochemistry (Moscow), 2006, 71 (1), S113S122

54. Monajjemi, M.; Yamola, H.; Mollaamin, F. Fullerenes, Nanotubes, and Carbon Nanostructures, 2014, 22, 595-603

55. Mollaamin, F.; Layali, I.; Ilkhani A. R.; Monajjemi, M. African Journal of Microbiology Research .2010, 4(24) 2795-2803

56. Mollaamin, F.; Shahani poor, p K. .; Nejadsattari, T. ; Monajjemi, M. African Journal of Microbiology Research. 2010, 4(20) 2098-2108

57. Monajjemi, M.; Ahmadianarog, M. Journal of Computational and Theoretical Nanoscience. 2014, 11(6), 1465-1471

58. Monajjemi, M.; Jafari Azan, M.; Mollaamin, F. Fullerenes, Nanotubes, and Carbon Nanostructures.2013, 21(6), 503-515

59. Mollaamin, F.; Monajjemi, M. Physics and Chemistry of Liquids .2012, 50, 5, 2012, 596-604

60. Monajjemi, M.; Khosravi, M.; Honarparvar, B.; Mollaamin, F.; International Journal of Quantum Chemistry, 2011, 111, 27712777

61. Khaleghian, M.; Zahmatkesh, M.; Mollaamin, 
F.; Monajjemi, M. Fullerenes, Nanotubes, and Carbon Nanostructures, 2011, 19(4): 251-261

62. Monajjemi, M.; Baheri, H.; Mollaamin, F. Journal of Structural Chemistry.2011 52(1), 54-59

63. Mahdavian, L.; Monajjemi, M.; Mangkorntong, N. Fullerenes, Nanotubes and Carbon Nanostructures, 2009, 17 (5), 484-495

64. Monajjemi, M., Mahdavian, L., Mollaamin, F. Bull. Chem. Soc. Ethiop. 2008, 22(2), 277286

65. Monajjemi, M.; Afsharnezhad, S, Jaafari, M.R..; Mirdamadi, S..; Mollaamin, F..; Monajemi, H. Chemistry .2008, 17 (1), 55-69

66. Monajjemi, M.; Mollaamin, F.; Gholami, M. R.; Yoozbashizadeh, H.; Sadrnezhaad, S.K.; Passdar, H.; Main Group Metal Chemistry, 2003, 26, 6, 349-361

67. Monajjemi, M.; Azad ,MT.; Haeri, HH.; Zare, K.; Hamedani, Sh.; JOURNAL OF CHEMICAL RESEARCH-S.2003, (8): 454-456

68. Monajjemi, M.; Najafpour, J. Fullerenes, Nanotubes, and Carbon Nanostructures, 2014, 22(6): 575-594

69. Monajjemi, M.; Noei, M.; Mollaamin, F. Nucleosides, Nucleotides and Nucleic Acids. 2010 29(9):676-683

70. Ghiasi, R.; Monajjemi, M. Journal of Sulfur Chemistry .2007, 28, 5, 505-511

71. Monajjemi, M.; Ghiasi, R.; Abedi, A. Russian Journal of Inorganic Chemistry.2005, 50(3), 382-388

72. Monajjemi, M. .; Naderi, F.; Mollaamin, F.; Khaleghian, M. J. Mex. Chem. Soc. 2012, 56(2), 207-211

73. 105. Monajjemi, M.; Farahani, N.; Mollaamin, F. Physics and Chemistry of Liquids, 2012, 50(2) 161-172

74. Monajjemi, M.; Seyed Hosseini, M. Journal of Computational and Theoretical Nanoscience .2013, 10 (10), 2473-2477

75. Monajjemi , M.; Honaparvar, B.; Khalili Hadad ,B.; Ilkhani ,AR.; Mollaamin, F. African Journal of Pharmacy and Pharmacology .2010, 4(8), $521-529$

76. Monajjemi, M. Theor Chem Acc, 2015, 134:77 DOI 10.1007/s00214-015-1668-9

77. Monajjemi, M. Journal of Molecular Modeling , 2014, 20, 2507
78. Monajjemi , M.; Honarparvar, B.; Monajemi, H.;. Journal of the Mexican Chemical Society, 2006, 50 (4), 143-148

79. Monajjemi, M.; Khaleghian, M.; Mollaamin, F. Molecular Simulation. 2010, 36, 11, 865-

80. Ilkhani, Ali R.; Monajjemi, M. Computational and Theoretical Chemistry.2015 1074, 19-25

81. Monajjemi, M. Biophysical Chemistry. 2015 207,114-127

82. Monajjemi, M., Moniri, E., Panahi, H.A , Journal of Chemical and Engineering Data.2001, 1249-1254.

83. Mollaamin, F.; Najafpour, J.; Ghadami, S.; Ilkhani, A. R.; Akrami, M. S.; Monajjemi, M. Journal of Computational and Theoretical Nanoscience. 11 (5), 1290-1298

84. Monajjemi, M.; Ghiasi, R.; Ketabi, S.; Passdar, H.; Mollaamin, F. Journal of Chemical Research . 2004, 1, 11.

85. Monajjemi, M.; Heshmat, M.; Haeri, H.H. Biochemistry (Moscow).2006, 71, 113-122

86. Monajjemi, M.; Heshmat, M.; Aghaei, H.;Ahmadi, R.; Zare, K. Bulletin of the Chemical Society of Ethiopia. 2007, 21, 111-116

87. Monajjemi, M., Kharghanian, L., Khaleghian, M., Chegini, H. Fullerenes Nanotubes and Carbon Nanostructures.2014, 22, 8, $0.1080 / 1536383 X .2012 .717563$

88. Sarasia, E.M.; Afsharnezhad, S.; Honarparvar, B.; Mollaamin, F.; Monajjemi, M. Physics and Chemistry of Liquids. 2011, 49 (5), 561-571

89. Amiri, A.; Babaeie, F.; Monajjemi, M. Physics and Chemistry of Liquids. 2008, 46, 4, 379389

90. Monajjemi, M.; Heshmat, M.; Haeri, H.H.; Kaveh, F. Russian Journal of Physical Chemistry A, 2006, 80, 7, 1061-1068

91. Monajjemi, M.; Moniri, E.; Azizi, Z.; Ahmad Panahi, H. Russian Journal of Inorganic Chemistry. 2005, 50, 1, 40-44

92. Jalilian,H.; Monajjemi, M. Japanese Journal of Applied Physics. 2015, 54, 8, 08510

93. Mollaamin, F.; Monajjemi, M. Journal of Computational and Theoretical Nanoscience. 2015, 12, 6, 1030-1039

94. Felegari, Z.; Monajjemi, M. Journal of Theoretical and Computational Chemistry. 2015, 14, 3, 1550021 
95. Shojaee, S., Monajjemi, M. Journal of Computational and Theoretical Nanoscience. 2015, 12, 3, 449-458

96. Esmkhani, R.; Monajjemi, M. Journal of Computational and Theoretical Nanoscience. 2015. 12, 4, 652-659

97. Monajjemi, M., Seyedhosseini, M., Mousavi, M., Jamali, Z. Journal of Computational and Theoretical Nanoscience. 2015 , 23 (3), 239244

98. Ghiasi, R.; Monajjemi, M.; Mokarram, E.E.; Makkipour, P. Journal of Structural Chemistry. 2008, 4 , 4, 600-605

99. Mahdavian, L.; Monajjemi, M. Microelectronics Journal. 2010, 41(2-3), 142-149

100. Monajjemi, M.; Baie, M.T.; Mollaamin, F. Russian Chemical Bulletin.2010, 59, 5, 886889

101. Bakhshi, K.; Mollaamin, F.; Monajjemi, M. Journal of Computational and Theoretical Nanoscience. 2011, 8, 4, 763-768

102. Darouie, M.; Afshar, S.; Zare, K., Monajjemi, M. journal of Experimental Nanoscience.2013, 8, 4, 451-461

103. Amiri, A.; Monajjemi, M.; Zare, K.; Ketabi, S. Physics and Chemistry of Liquids. 2006, 44, 4, 449-456.

104. Zonouzi, R.;Khajeh, K.; Monajjemi, M.; Ghaemi, N. Journal of Microbiology and Biotechnology. 2013, 23, 1 ,7-14

105. Ali R. Ilkhani .; Majid Monajjemi, Computational and Theoretical Chemistry.2015, 1074 19-25

106. Tahan, A.; Mollaamin, F.; Monajjemi, M. Russian Journal of Physical Chemistry A, 2009, 83 (4), 587-597

107. Khalili Hadad, B.; Mollaamin, F.; Monajjemi, M, Russian Chemical Bulletin,2011, 60(2):233236

108. Mollaamin, F.; Monajjemi, M.; Salemi, S.; Baei, M.T. Fullerenes Nanotubes and Carbon Nanostructures, 2011, 19, 3, 182-196

109. Mollaamin, F.; Shahani Pour.; K., Shahani Pour, K.; ilkhani, A.R.; Sheckari, Z., Monajjemi, M Russian Chemical Bulletin, 2012 , 61(12), 2193-2198

110. Shoaei, S.M.; Aghaei, H.; Monajjemi, M.; Aghaei, M. Phosphorus, Sulfur and Silicon and the Related Elements. 2014, 189, 5; 652660
111. Mehrzad, J., Monajjemi, M., Hashemi, M , Biochemistry (Moscow).2014, 79 (1), 31-36

112. Moghaddam, N.A., Zadeh, M.S., Monajjemi, M. Journal of Computational and Theoretical Nanoscience , 2015 , Vol. 12, No. 3, doi:10.1166/jctn.2015.3736

113. Joohari, S.; Monajjemi, M , Songklanakarin Journal of Science and Technology , 2015 , 37(3):327

114. Rajaian, E., Monajjemi, M., Gholami, M.R, Journal of Chemical Research - Part S, 2002, 6, 1, 279-281

115. Ghassemzadeh, L., Monajjemi, M., Zare, K, Journal of Chemical Research - Part S, 2003, 4, 195-199

116. Mehdizadeh Barforushi,M.; Safari,S.; Monajjemi,M. J. Comput. Theor. Nanosci.2015, 12, 3058-3065.

117. Mollaamin,F.; Ilkhani,A.; Sakhaei,N.; Bonsakhteh,B.; Faridchehr,A.; Tohidi,S.; Monajjemi, M. J. Comput. Theor. Nanosci. 2015,12, 3148-3154

118. Rahmati,H.; Monajjemi,M. J. Comput. Theor. Nanosci. 2015, 12, 3473-3481.

119. Roghieh Tarlani Bashiz,R.; Monajjemi,M. J. Comput. Theor. Nanosci.2015, 12, 38083816

120. Mehrabi Nejad,A.; Monajjemi,M. J. Comput. Theor. Nanosci. 2015, 12, 3902-3910.

121. Monajjemi,M.; Bagheri,S.; Moosavi,M.S.; Moradiyeh,N.; Zakeri,M.;Attarikhasraghi,N.; Saghayimarouf,N.; Niyatzadeh,G.; Shekarkhand,M.; Khalilimofrad,M.S.; Ahmadin,H.; Ahadi,M.; Molecules 2015, 20, 21636-21657; doi:10.3390/ molecules201219769

122. Shabanzadeh,E.; Monajjemi,M.; J. Comput. Theor. Nanosci.2015, 12, 4076-4086

123. Elsagh,A.; Jalilian, H.; Kianpour, E.; Ghazi, Mokri, H.S.; Rajabzadeh,M.; Moosavi,M.S.; Ghaemi Amiri,F.; Majid Monajjemi ,M.; J. Comput. Theor. Nanosci. 2015, 12, 4211 4218.

124. Faridchehr, A.; Rustaiyan,A.; Monajjemi,M.; J. Comput. Theor. Nanosci.2015, 12, 4301 4314.

125. Tohidi, S.; Monajjemi, M.; Rustaiyan, A.; J. Comput. Theor. Nanosci. 2015, 12, 43454351

126. Ali Akbari Zadeh,M.; Lari,H.; Kharghanian,L.; 
Balali,E.; Khadivi,R.;Yahyaei,H.; Mollaamin,F.; Monajjemi, M.; J. Comput. Theor. Nanosci.2015, 12, 4358-4367.

127. Dezfooli,S.; Lari,H.; Balali,E.; Khadivi,R.; Farzi,F.; Moradiyeh,N.; Monajjemi,M.; J. Comput. Theor. Nanosci.2015, 12, 4478-4488

128. Jalilian,H.; Sayadian,M.; Elsagh,A.; Farzi,F.; Moradiyeh,N.; Samiei Soofi,N.; Khosravi,S.;. Mohammadian,N.T.; Monajjemi,M. J. Comput. Theor. Nanosci. 2015,12, 4785-4793.

129. Farzi,F.; Bagheri,S.; Rajabzadeh,M.; Sayadian,M.; Jalilian,H.; Moradiyeh,N.; Monajjemi, M.; J. Comput. Theor. Nanosci.2015, 12, 4862-4872.

130. Monajjemi,M.; Mohammadian, N.T, J. Comput. Theor. Nanosci. 2015, 12, 4895-4914

131. Monajjemi, M., Chahkandi, B. Journal of Molecular Structure: THEOCHEM, 2005, 714 (1), 28, 43-60.
132. Wang, B.; Zhao,X.; Wang,J. ; Guo,H. Appl. Phys. Lett, 1999, 74 ,2887-2889.

133. Wang, J.; et al., Phys. Rev. Lett., 1998, 80 , 4277-4280.

134. Zhao,Y.; Truhlar,D.G.; Theor Chem Account,2008, 120 , 215-241.

135. Zhao,Y.; Truhlar,D.G.; Accounts of Chemical Research,2008, 41(2), 157-167.

136. Check,C.E.; Gilbert,T.M.; J. Org. Chem ,2005,70, 9828-9834.

137. Ao , Z.; Yang,J.; S.Li.; Jiang Q.; ChemPhys Lett ,2008, 461(4), 276-279.

138. Kohn, W. ; Sham, L. J. Phys. Rev. 1965,140 A , 1133-1138.

139. Perdew, J.P. ; Burke, K. Ernzerhof, Phys. Rev. Lett. 1996.77, 3865-3868.

140. Blöchl, P. E. O. Jepsend, O. K. Andersen, Phys. Rev. B, 1994; 49, 16223. 